\title{
Aquaporin 4 and neuromyelitis optica
}

\author{
Dr Marios C Papadopoulos, MD and Prof A S Verkman, MD \\ Academic Neurosurgery Unit, St George's, University of London, London, UK (Dr M C \\ Papadopoulos MD); and Department of Medicine and Department of Physiology, University of \\ California San Francisco, San Francisco, CA, USA (Prof A S Verkman MD)
}

\begin{abstract}
Neuromyelitis optica is an inflammatory demyelinating disorder of the CNS. The discovery of circulating IgG1 antibodies against the astrocyte water channel protein aquaporin 4 (AQP4) and the evidence that AQP4-IgG is involved in the development of neuromyelitis optica revolutionised our understanding of the disease. However, important unanswered questions remain-for example, we do not know the cause of AQP4-IgG-negative disease, how astrocyte damage causes demyelination, the role of T cells, why peripheral AQP4-expressing organs are undamaged, and how circulating AQP4-IgG enters neuromyelitis optica lesions. New drug candidates have emerged, such as aquaporumab (non-pathogenic antibody blocker of AQP4-IgG binding), sivelestat (neutrophil elastase inhibitor), and eculizumab (complement inhibitor). Despite rapid progress, randomised clinical trials to test new drugs will be challenging because of the small number of individuals with the disorder.
\end{abstract}

\section{Introduction}

The coexistence of optic neuritis with myelitis was recognised in the early 19th century by several physicians, such as the Frenchman Antoine Portal, the Italian Giovanni Battista Pescetto, the Englishman Lockhart Clarke, and the Scotsman John Abercrombie. ${ }^{1-3}$ Abercrombie described intractable vomiting in some of these patients, indicating involvement of the medulla. In 1894, the French neurologist Eugène Devic and his student Fernand Gault reviewed published cases of optic neuritis with myelitis, after which the disease became known as neuromyelitis optica or Devic's disease. ${ }^{1-3}$

Neuromyelitis optica was thought to be a variant of multiple sclerosis, but in 2004, a circulating IgG auto-antibody was reported in patients with neuromyelitis optica that was absent in those with multiple sclerosis. ${ }^{4}$ Within a year, the astrocyte water channel protein aquaporin 4 (AQP4) was identified as its target. ${ }^{5}$ Here, we review advances in understanding of the pathogenesis of neuromyelitis optica and their implications for clinical practice. We explain the structure, function, and biological roles of AQP4, and the cellular effects of AQP4-IgG binding. We then summarise the evidence that AQP4- $\operatorname{IgG}$ causes disease and discuss major unanswered questions. We refer readers elsewhere ${ }^{6-8}$ for reviews with a clinical focus, including diagnosis and present treatments.

Correspondence to: Dr Marios C Papadopoulos, Academic Neurosurgery Unit, Room 1.122 Jenner Wing, St George's, University of London, London SW17 0RE, UK, mpapadop@sgul.ac.uk.

Contributors

MCP and ASV searched for published work and wrote the Review.

Conflicts of interest

The University of California San Francisco and the University of Colorado have filed a joint US provisional patent application for aquaporumab antibody treatment for neuromyelitis optica, of which ASV is a named co-inventor. The intellectual property rights are owned by the University of California and the University of Colorado. MCP declares that he has no conflicts of interest. 


\section{Epidemiology}

A definitive diagnosis of neuromyelitis optica can be made when optic neuritis, myelitis, and at least two of three supportive criteria (MRI evidence of a contiguous spinal cord lesion in 23 segments; brain MRI not diagnostic of multiple sclerosis; and AQP4-IgG seropositivity) are present. ${ }^{7}$ This scheme allows for a diagnosis of neuromyelitis optica in some patients with brain lesions who would not be thought to have the disorder, and when detectable AQP4-IgG is absent.

The spectrum of neuromyelitis optica disorders encompasses definitive neuromyelitis optica, as well as limited forms, such as AQP4-IgG-positive, longitudinally extensive, transverse myelitis, or recurrent or bilateral AQP4-IgG-positive optic neuritis. The frequency of AQP4IgG seropositivity is higher in patients with relapsing disease, optic neuritis, or longitudinally extensive transverse myelitis than in those with monophasic disease. ${ }^{7-9}$ The percentage of patients with demyelinating disease that fulfil the present diagnostic criteria is low (1-2\%) in white people from Europe, North America, or Australia, and high (20-48\%) in people from the West Indies and Asia. ${ }^{8}$

The prevalence of neuromyelitis is estimated at $0 \cdot 3-4 \cdot 4$ per 100000 individuals, ${ }^{8}$ equivalent to $900-13200$ people in the USA and $180-2640$ in the UK. This prevalence is likely to increase as AQP4-IgG testing becomes wide spread and AQP4-IgG assay sensitivity improves. The median age at presentation is 39 years. ${ }^{8}$ Women are most commonly affected, accounting for roughly $85 \%$ of cases. ${ }^{8}$ Occurrence of relapse might increase in the last trimester of pregnancy and post partum, ${ }^{10}$ and safe prophylactic treatment might be appropriate during late pregnancy and breastfeeding. Transmission of neuromyelitis optica from mother to fetus has not been reported, possibly because little AQP4 is expressed in the fetal CNS before formation of the blood-brain barrier. ${ }^{11}$ An intriguing association between the disorder and myasthenia gravis has been noted, with neuromyelitis optica often developing decades after myasthenia. ${ }^{12,13}$

About 3\% of patients with neuromyelitis optica have relatives with the disease, but the underlying genetic susceptibility is complex. ${ }^{14}$ Some HLAs are associated with increased risk of neuromyelitis, such as DRB $1 * 0301$ in white people and people with one white and one black parent, ${ }^{15}$ and DPB $1 * 0501$ in people from Asia. ${ }^{16}$ The HLA associations are different in multiple sclerosis compared with neuromyelitis optica. ${ }^{15,16}$ Genetic variations in the AQP4 sequence do not substantially account for neuromyelitis optica susceptibility. ${ }^{17,18}$

\section{AQP4: the target of antibodies}

\section{Structure, function, and cellular localisation}

AQP4 was originally cloned from rat lung in 1994, on the basis of its homology with other aquaporins. ${ }^{19} \mathrm{~A}$ high-resolution $\mathrm{x}$-ray structure shows that AQP4 monomers consist of six helical, membrane-spanning domains and two short helical segments surrounding a narrow aqueous pore (figure 1), ${ }^{20}$ similar to other aquaporins. Additionally, as with other aquaporins, $\mathrm{AQP} 4$ monomers assemble as tetramers; however, $\mathrm{AQP} 4$ tetramers uniquely further aggregate in cell plasma membranes to form supramolecular assembles called orthogonal arrays of particles (OAPs). OAPs were originally visualised in membranes by freeze-fracture electron microscopy (figure 1). ${ }^{21} \mathrm{AQP} 4$ was identified as the major OAP protein from the appearance of OAPs in AQP4-transfected cells ${ }^{22}$ and their absence in mice that did not have AQP4. ${ }^{23}$ Advanced optical microscopy methods have elucidated OAP dynamics ${ }^{24}$ and regulatory mechanisms. ${ }^{25,26}$ Of relevance to neuromyelitis optica, most AQP4-IgGs bind preferentially to OAPs; $; 9,27$ OAPs seem to be crucial for complementdependent cytotoxicity through multivalent $\mathrm{C} 1 \mathrm{q}$ binding to clustered AQP4-IgG. ${ }^{28}$ 
AQP4 is present in two major isoforms produced by alternative splicing: a relatively long (M1) isoform with translation initiation at Met-1, and a shorter (M23) isoform with translation initiation at Met-23 (figure 1). Both isoforms are expressed in astrocytes and comingle to form heterotetramers. M23-AQP4 forms OAPs because of intermolecular Nterminus interactions with residues just downstream of Met-23; M1-AQP4 does not form OAPs on its own and restricts the size of OAPs when coexpressed with M23-AQP4. ${ }^{25,29,30}$ Both M1-AQP4 and M23-AQP4 function as bidirectional, water-selective channels that enable water flow across cell membranes in response to osmotic gradients.

AQP4 is most strongly expressed in the CNS, but is also present in epithelial cells in the kidney (collecting duct), the stomach (parietal cells), airways, glands, and skeletal muscle. ${ }^{31}$ AQP4 is expressed in astrocytes in the brain, spinal cord, and optic nerve, and is particularly concentrated at pial and ependymal surfaces in contact with the CSF. ${ }^{32,33}$ At the cell level, AQP4 expression is polarised to foot processes of astrocytes in contact with blood vessels. ${ }^{32,33} \mathrm{AQP} 4$ is also expressed in so-called supportive cells-similar to astrocytes-in sensory organs such as Müller cells in the retina. ${ }^{34}$ Pathological changes associated with neuromyelitis optica mostly occur in the spinal cord and optic nerve, and to a lesser extent in the brain, with a notable absence of abnormalities in peripheral AQP4-expressing tissues.

\section{Biological roles}

Knowledge of the biological roles of AQP4 is important because AQP4 expression is reduced in neuromyelitis optica lesions as a result of astrocyte cytotoxicity. Available information about AQP4 physiology comes mainly from AQP4 knockout mice because people with loss-of-function mutations in AQP4 have not been identified. At baseline, AQP4 knockout mice have normal appearance, survival, growth, ${ }^{35}$ and neuromuscular function, ${ }^{36}$ but partial impairment in visual, ${ }^{34}$ auditory, ${ }^{37}$ and olfactory ${ }^{38}$ signalling, as deduced from electrophysiological or behavioural studies, or both.

Several phenotypes of AQP4 knockout mice have been identified after various stresses have been imposed. Outside of the CNS, AQP4 knockout mice have a mild reduction in maximum urine concentration, which is related to reduced water permeability in the renal inner medullary collecting duct. ${ }^{35}$ In the brain, mice without AQP4 show reduced cytotoxic (cell swelling) brain oedema after water intoxication or ischaemic stroke, ${ }^{39}$ increased vasogenic (leaky vessel) brain oedema in brain tumours, ${ }^{40}$ and increased ventricular enlargement in obstructive hydro cephalus. ${ }^{41}$ These phenotypes can be explained by bidirectional impairment in water transport with AQP4 deficiency, with reduced water entry into the brain in cytotoxic oedema and impaired removal of excess interstitial water in vasogenic oedema. In the spinal cord, swelling is reduced and neurological outcome is improved in AQP4 knockout mice in a model of cytotoxic oedema (transient cord compression), ${ }^{42}$ but worsened in a model of vasogenic oedema (contusion injury). ${ }^{43}$

Phenotype studies ${ }^{31,44-46}$ have implicated AQP4 in neuroexcitation, astrocyte migration, and neuroinflammation. AQP4 knockout mice have in creased seizure duration, ${ }^{44}$ impaired glial scarring, ${ }^{45}$ and attenuated experimental autoimmune encephalo myelitis. ${ }^{46}$ Impaired AQP4 water permeability in astrocytes is probably the cause of these phenotypes. Cellular mechanisms of AQP4-dependent astrocyte migration, neurosensory signalling, and inflammation have been reviewed previously. ${ }^{31}$

\section{AQP4-IgG binding to AQP4}

Several studies $9,27,47,48$ have shown that more binding of AQP4-IgG in the serum of patients with neuromyelitis optica occurs with transfected cells expressing M23-AQP4 than with M1-AQP4. This difference might be caused by AQP4-IgG preferentially binding to OAPs, 
because M23-AQP4 forms OAPs and M1-AQP4 does not. Binding specificity has been investigated with a two-colour quantitative fluorescence ratio imaging assay of AQP4transfected astrocyte cells stained with AQP4-IgG and a C-terminus anti-AQP4 antibody. ${ }^{27}$ The researchers used serum from control patients and those with neuromyelitis optica, and monoclonal recombinant AQP4-IgGs derived from clonally expanded plasma cells from the CSF of individuals with seropositive neuromyelitis optica. ${ }^{49}$ Different monoclonal and serum AQP4-IgGs showed wide variation in binding to M1-AQP4 or M23-AQP4, from similar binding to both isoforms to exclusive binding to M23-AQP4. The affinity of the tightest binding AQP4-IgG was $44 \mathrm{nM} .^{27}$

Measurements in cells expressing M23-AQP4 with OAP-disrupting mutations suggested that the differential binding of AQP4-IgG to M1 or M23 is due to OAP assembly; studies ${ }^{27}$ with purified Fab fragments suggested that the AQP4 epitope undergoes a structural change on array assembly. Some reports ${ }^{50,51}$ have shown that specific extracellular epitopes of AQP4 might be associated with AQP4-IgG binding; however, the data are difficult to interpret because polyclonal AQP4-IgG was studied, and the AQP4-IgG binding site probably includes three-dimensional epitopes that are specific to AQP4-IgG and are potentially modified by point mutations in AQP4 extracellular loops. Although clinical AQP4-IgG assays give semi-quantitative titres to one AQP4 isoform or to both isoforms, ${ }^{52}$ measurement of concentration-dependent binding to M1-AQP4 and M23-AQP4 separately might provide useful information about how to monitor and manage clinical neuromyelitis optica.

\section{Cellular effects of AQP4-IgG binding to AQP4}

Antibodies can have several effector functions when bound to their target: modification of target function; target internalisation, reducing surface expression; complement activation to cause cell death (complement-dependent cytotoxicity); and activation of effector cells, such as natural killer cells, to cause cell death (antibody-dependent cell-mediated cytotoxicity). Although one investigation with frog oocytes suggested that AQP-IgG binding causes partial inhibition of AQP4 water permeability, ${ }^{48}$ another with cultured rat cortical astrocytes ${ }^{47}$ and other work ${ }^{53}$ showed no effect: the large size of AQP4-IgG compared with AQP4 precludes binding of more than one antibody per AQP4 tetramer.

Initial studies with AQP4-transfected HEK293 cells ${ }^{48,54}$ showed that AQP4-IgG binding causes AQP4 internalisation; however, there was little or no internalisation in mouse cortical astrocyte cultures or mouse brain in vivo after binding. ${ }^{55}$ In mice and in vitro, AQP4-IgG binding to AQP4 causes complement-dependent cytotoxicity when complement is present, ${ }^{54,56}$ and antibody-dependent cell-mediated cytotoxicity when natural killer cells are present. ${ }^{57-59}$ Complement-dependent cytotoxicity is probably the major mechanism by which neuromyelitis optica develops. ${ }^{56,60-62}$

\section{Mechanisms of pathogenesis}

\section{Overview}

Substantial evidence is available that AQP4-IgG initiates formation of a neuromyelitis optica lesion once it enters the CNS (figure 2). Complement-mediated astrocyte damage occurs first, followed by granulocyte infiltration, oligodendrocyte death, and ultimately neuronal cell death. Because the lesions are characterised by necrosis of the major CNS cell types, ${ }^{63}$ the clinical deficits are often severe and recovery is poor. ${ }^{8} \mathrm{AQP} 4-\mathrm{IgM}$ is found in $10 \%$ of serum samples positive for AQP4-IgG, but its role in the disease remains unknown. ${ }^{64}$ 


\section{Evidence that AQP4-IgG is pathogenic}

AQP4-IgG binds to AQP4 on the plasma membrane of non-permeabilised cells, such as AQP4-transfected HEK293 and Chinese hamster ovary cells, and mouse cortical astrocyte cultures. ${ }^{54-56}$ Because AQP4-IgG is an IgG1-isotype antibody, bound AQP4-IgG causes complement-dependent cytotoxicity. Exposure of AQP4-transfected HEK293 and Chinese hamster ovary cells, and mouse cortical astrocytes to AQP4-IgG and complement causes plasma membrane deposition of the complement membrane attack complex and cytotoxicity. ${ }^{54,56} \mathrm{AQP} 4-\mathrm{IgG}$ also causes antibody-dependent cell-mediated cytotoxicity of mouse cortical astrocyte cultures when natural killer cells are present in the absence of complement. ${ }^{57,59}$ These findings provide evidence for AQP4-IgG pathogenicity at the cell level.

Evidence from clinical observations ${ }^{6,65}$ and histological and animal studies ${ }^{61-63}$ also shows that AQP4-IgG causes neuromyelitis optica lesions. AQP4-IgG is identified in most patients with the disease (68-91\%) and is highly specific (85-99\%). ${ }^{65}$ Generally, AQP4-IgG serum concentrations increase before a relapse, ${ }^{65}$ and a decrease in plasma AQP4-IgG by plasmapheresis reduces disease activity. ${ }^{6}$

Human neuromyelitis optica lesions show vasculocentric deposition of immunoglobulin and activated complement components, suggesting humoral immunity against a perivascular antigen. ${ }^{61-63}$ The substantial loss of AQP4 and the astrocyte marker glial fibrillary acidic protein (GFAP) in lesions is consistent with a humoral response targeting AQP4 and causing astrocyte death. The presence of a few AQP4-negative or GFAP-positive astrocytes in lesions suggests that loss of AQP4 precedes astrocyte death. ${ }^{61-63}$ Occasionally, AQP4 and GFAP are lost, but myelin is preserved, ${ }^{61-63}$ supporting the notion that astrocyte death precedes myelin breakdown.

Granulocytes (neutrophils and eosinophils) and macrophages predominate in human neuromyelitis optica lesions, with a paucity of T lymphocytes ${ }^{63}$ and natural killer cells (unpublished). Most neutrophils are degranulated (figure 2) ${ }^{66}$ with evidence from mouse models $^{66,67}$ and case reports ${ }^{68}$ implicating neutrophils early in lesion development. The presence of eosinophils, which are characteristically seen in allergic and parasitic ${ }^{69}$ rather than antibody-mediated diseases, is intriguing. In neuromyelitis optica, the eosinophils might stimulate the humoral immune response by producing type 2 cytokines, and might contribute to CNS damage by secreting eosinophil-derived neurotoxin and generating free radicals. ${ }^{69}$

Lesions similar to those reported in neuromyelitis optica have been produced in rodents by passive transfer of AQP4-IgG. ${ }^{46,56,70,71}$ Intraperitoneal injection of AQP4-IgG exacerbates CNS inflammation in rats with experimental autoimmune encephalomyelitis ${ }^{49,70}$ or those pretreated with Freund's adjuvant. ${ }^{71}$ Although these studies provide evidence that AQP4$\mathrm{IgG}$ is pathogenic, the pre-existing inflammation confounds further interpretation. Direct evidence that AQP4-IgG is pathogenic in vivo comes from intracerebral injection of AQP4$\operatorname{IgG}$ and human complement in mice, which reproduces the characteristic histological features of human neuromyelitis optica lesions, such as loss of AQP4 and GFAP, inflammatory cell infiltration, loss of myelin, and perivascular deposition of activated complement. ${ }^{56}$ In these experiments, human complement is injected because AQP4-IgG does not activate mouse complement effectively. The cell infiltrate consists of granulocytes and macrophages with few T lymphocytes, similar to human lesions. In key control experiments, no lesions are produced when complement is injected with $\mathrm{IgG}$ from individuals without neuromyelitis optica or with AQP4-IgG-depleted neuromyelitis optica serum, when complement is inhibited or not administered, or when complement and AQP4$\mathrm{IgG}$ are injected in AQP4-deficient mice. The intracerebral injection mouse model does not 
reproduce clinical signs of neuromyelitis optica because of the site of injection. An ex-vivo model of neuromyelitis optica has been described, ${ }^{72}$ in which exposure of cultured mouse spinal cord slices or optic nerve to AQP4-IgG and complement causes loss of AQP4, GFAP, and myelin.

\section{Origin of lesions}

AQP4-IgG is more than 500 times more concentrated in plasma than in CSF, which has been taken to suggest that AQP4-IgG forms peripherally and enters the CNS secondarily. ${ }^{73}$ However, plasma cells that secrete AQP4-IgG have been detected in the $\mathrm{CSF}^{49}$ and could potentially lodge in CNS tissue and constitute a pathogenically relevant source of AQP4IgG production. Circulating AQP4-IgG alone is insufficient to produce neuromyelitis optica lesions, because serial plasma samples obtained from patients in remission often have AQP4-IgG, as do serum samples taken before disease becomes clinically apparent. ${ }^{65}$

Substantial evidence from rodent models ${ }^{56,58,66,70}$ suggests that, once in the CNS, AQP4IgG causes astrocyte cytotoxicity, initially by complement activation and then by a cascade of inflammatory events such as granulocyte infiltration and then macrophage infiltration, causing further disruption of the blood-brain barrier (figure 2). The central involvement of astrocyte damage has prompted assays for astrocytic markers, such as CSF GFAP, for monitoring of disease progression and response to treatment. ${ }^{74}$ Studies in humans ${ }^{62,63,66,67,75,76}$ support a key role for neutrophils and eosinophils in neuromyelitis optica. Granulocytes are abundant within lesions, ${ }^{62,63,66}$ and patients often have granulocytes ${ }^{66,76}$ and high concentrations of granulocyte colony-stimulating factor in their $\mathrm{CSF},{ }^{76}$ and a serum granulocyte cytokine signature.${ }^{67}$

The role of macrophages, which abundantly infiltrate lesions (figure 2), is unclear. In multiple sclerosis, activated macrophages cause axonal damage by phagocytosing myelin and secreting proinflammatory cytokines, free radicals, glutamate, and metalloproteases. ${ }^{77}$ Macrophages could scavenge the cellular debris produced by astrocyte cytotoxicity and granulocyte infiltration. ${ }^{77}$ The detrimental effect of natalizumab, a monoclonal anti-a4integrin antibody, in some patients with neuromyelitis optica ${ }^{78,79}$ suggests that macrophages potentially have a beneficial role in the disorder. a4-Integrin is expressed in human macrophages but not in neutrophils, ${ }^{80}$ and so would inhibit macrophage but not neutrophil entry into lesions.

Demyelination, a characteristic feature of human and mouse neuromyelitis optica, is not a primary event. Mouse experiments show that myelin loss is a result of astrocyte damage ${ }^{81}$ and leads to axonal degeneration ${ }^{56}$ (figure 2 ). In humans ${ }^{63}$ and mouse models, ${ }^{56}$ neuronal necrosis follows axonal degeneration (figure 2).

\section{Unanswered questions and controversies}

\section{What causes AQP4-IgG-negative disease?}

Whether AQP4-IgG-negative neuromyelitis optica differs from AQP4-IgG-positive disease is debated. Reports show that AQP4-IgG-positive neuromyelitis optica differs clinically and epidemiologically from seronegative disease (strong predominance in women; frequent association with coexisting autoimmunity; more severe clinical attacks; higher spinal cord lesion load; and more frequent relapse) $)^{65,82}$ and that the HLA susceptibility profile of AQP4-IgG-negative disorder could be more similar to that of multiple sclerosis than of AQP4-positive neuromyelitis optica. ${ }^{15}$

Another possibility is that $\mathrm{AQP} 4-\mathrm{IgG}$ is present in some patients with seronegative disease, but present assays are not sensitive enough to detect it. The proportion of patients with 
AQP4-IgG-seronegative neuromyelitis optica who have AQP4-IgG in the CSF is low. ${ }^{73,83}$ With improvements in assay sensitivity, serum AQP4-IgG has been detected in an increasing number of patients with apparently AQP4-IgG-negative disease ${ }^{84}$ Some patients with AQP4-IgG-negative neuromyelitis optica might have antibodies against antigens other than AQP4, perhaps against other astrocyte targets. IgG1 antibodies that can mediate complement-dependent cytotoxicity against myelin oligodendrocyte glycoprotein have been detected in some patients with AQP4-IgG-negative disease, and in some with recurrent optic neuritis, longitudinally extensive transverse myelitis, multiple sclerosis, or lupus. ${ }^{85}$ Together, these findings suggest that AQP4-IgG-negative disease is probably a heterogeneous group that could include some patients with multiple sclerosis, some who are AQP4-IgG-positive, some with other autoantibodies, and perhaps some whose disease is not antibody mediated.

\section{How does AQP4-IgG enter the CNS?}

Unlike other CNS autoimmune diseases, neuromyelitis optica is notable for the absence of intrathecal antibody synthesis. ${ }^{73,75}$ Serum AQP4-IgG might be present for years before onset of the disorder ${ }^{86}$ Disease in the brain could start with the movement of AQP4-IgG from the periphery into the circumventricular organs, which do not have a blood-brain barrier but express AQP4. The involvement of the area postrema-causing nausea, vomiting and hiccups ${ }^{87}$ —and of the posterior hypothalamus—causing inappropriate antidiuretic hormone secretion-supports this notion. ${ }^{88}$

The reason why pathological changes occur in the optic nerves and spinal cord is unclear. The blood-brain barrier might not be fully developed in the prelaminar portion of optic nerve ${ }^{89}$ and root entry zones in the spinal cord, ${ }^{90}$ allowing easier access of circulating IgG than elsewhere in the CNS. Perhaps an additional factor, such as an infection, transiently disrupts the blood-brain barrier preferentially in the optic nerve or spinal cord, allowing AQP4-IgG access, as suggested by reports that $25-30 \%$ of exacerbations are preceded by infections. ${ }^{91,92}$ Downregulation of the neonatal Fc receptor ${ }^{93}$ and disruption of the AQP4rich interfaces between the peripheral and central nervous systems ${ }^{94}$ have not been investigated. The population of circulating plasmablasts increases before an episode of neuromyelitis optica and they migrate into the $\mathrm{CNS},{ }^{95}$ raising the possibility of AQP4-IgG production by plasmablasts in the CNS. Exposure of plasmablasts to interleukin-6 (IL-6), which is high in serum and CSF of patients with neuromyelitis optica, promotes AQP4-IgG production. ${ }^{95}$ Therefore, research into inhibition of IL-6 receptors with tocilizumab in neuromyelitis optica might be worthwhile.

\section{Does AQP4-IgG damage peripheral AQP4-expressing organs?}

Although peripheral AQP4-expressing organs do not have the equivalent of the blood-brain barrier and were shown in a mouse model ${ }^{96}$ to rapidly bind circulating AQP4-IgG, no substantial peripheral organ damage occurs in neuromyelitis optica. One study ${ }^{97}$ showed that serum creatine kinase concentrations were raised in less than $0.5 \%$ of patients, indicating possible skeletal muscle damage. Potential explanations for the absence of substantial abnormalities are a higher expression of M1-AQP4 than of M23-AQP4 in the peripheral nervous system, the minimal importance of peripheral AQP4-expressing cells for tissue function compared with astrocytes for CNS function, ${ }^{35,36,97}$ the low AQP4 expression in the peripheral nervous system compared with the CNS, ${ }^{98}$ and the unique extracellular microenvironment in some peripheral organs (eg, gastric acidity reducing AQP4-IgG binding). However, these proposed explanations are not supported by the known biochemistry and physiology of AQP4. ${ }^{96}$ 
Another possibility is reduced expression of complement regulator proteins (CD46, CD55, CD59) ${ }^{99}$ in astrocyte foot processes compared with peripheral AQP4-expressing cells, causing AQP4-expressing cells in the peripheral nervous system to have increased resistance to complement-dependent cytotoxicity. The absence of pathological changes in peripheral organs of patients with neuromyelitis optica might also reflect an absence of components of the neuroinflammatory cascade in these tissues (eg, microglia) and confinement of inflammatory cells and cytokines to the small extracellular space in the CNS.

\section{How does astrocyte damage cause myelin loss?}

Ablation of reactive astrocytes in mice by genetic manipulation causes substantial leucocyte infiltration and myelin loss after traumatic CNS injury, ${ }^{100}$ suggesting important interactions exist between astrocytes and oligodendrocytes. Gap junctional connections ${ }^{101}$ could propagate the astrocyte damage signals to oligodendrocytes. Additionally, AQP4 might colocalise with the excitatory aminoacid transporter EAAT2 in astrocytes, and EAAT2 downregulation in neuromyelitis optica might cause glutamate excitotoxicity and oligodendrocyte damage. ${ }^{102}$ However, the involvement of EAAT2 in neuromyelitis optica pathogenesis has been questioned because little or no internalisation of EAAT2 occurs in primary astrocyte cultures, glutamate uptake is unaffected by AQP4-IgG exposure, and intracerebral injection of AQP4-IgG in mice in vivo does not cause internalisation of AQP4 or AQP4-IgG. ${ }^{55}$

Another possibility is that reactive astrocytes initiate a positive-feedback cycle, resulting in widespread damage to several cell types, such as oligodendrocytes. Any CNS lesion generates AQP4-expressing reactive astrocytes in the surrounding CNS, which migrate towards the lesion. ${ }^{45,103}$ This finding also applies in neuromyelitis optica, in which many AQP4-expressing reactive astrocytes surround lesions in people ${ }^{61,63}$ and mice. ${ }^{56}$ In CNS lesions not associated with the disorder, reactive astrocytes enter the lesions to form a glial scar, but in neuromyelitis optica only a few scattered reactive astrocytes are present in the lesions. ${ }^{61}$ We suggest that the reactive astrocytes are promptly destroyed as they enter a neuromyelitis optica lesion, because of exposure to AQP4- $\operatorname{IgG}$ and complement. Thus, the entry of reactive astrocytes in such a lesion might sustain damage, leading to oligodendrocyte damage and demyelination.

\section{What roles do $\mathrm{T}$ cells have?}

T cells are implicated in neuromyelitis optica, because AQP4-IgG is a T-cell-dependent immunoglobulin subclass (IgG1). T cells are probably involved in the peripheral immune response, including breaking tolerance (when the immune system becomes responsive to self antigens) and antibody production. Indirect evidence from models of experimental autoimmune encephalomyelitis has suggested that $\mathrm{T}$ cells could cause neuromyelitis optica inflammation to be targeted to the optic nerve and spinal cord. ${ }^{70}$ Findings that suggest that $\mathrm{T}$ cells are probably not involved directly in lesion formation include: the high plasma versus low CSF AQP4-IgG titre; ${ }^{104}$ the paucity of T cells in human ${ }^{63}$ and mouse ${ }^{105}$ lesions; the harmful effect of natalizumab (which inhibits CNS entry of leucocytes, including T cells, but not neutrophils) in some patients with neuromyelitis optica; ${ }^{78,79}$ the ability to produce similar lesions in nude (T-cell-depleted) mice; ${ }^{105}$ and the development of widespread lesions in patients after inhibition of T-cell entry into the CNS with fingolimod. ${ }^{106}$ Th17 cells, a subgroup of $\mathrm{T}$ helper cells, might be involved in the recruitment of granulocytes into early lesions by inducing release of cytokines, such as CXCL8, from non-T cells. ${ }^{67}$ The generation of AQP4-specific T cells ${ }^{107}$ might help to clarify the role of T cells in rodent models of neuromyelitis optica. 


\section{Treatment strategies}

\section{Present treatments}

Acute neuromyelitis optica is treated with methyl-prednisolone and, in resistant cases, plasmapheresis. ${ }^{6}$ Maintenance treatments are corticosteroids, azathioprine, rituximab, mitoxantrone, cyclophosphamide, and mycophenolate ${ }^{6}$ Treatment regimens vary widely, ranging from corticosteroids alone to complex drug combinations. The rarity of neuromyelitis optica has precluded large-scale, randomised trials to rationalise treatment.

Interferon beta-the most commonly used therapy for multiple sclerosis-is acknowledged to be generally harmful in neuromyelitis optica. It increases relapses and AQP4-IgG antibody titre, ${ }^{108,109}$ probably by exacerbating Th17-mediated inflammation and demyelination, ${ }^{67}$ and by increasing the amounts of B-cell activating factor. ${ }^{110}$ Reports ${ }^{78-80}$ have suggested that natalizumab, which is also used for treating multiple sclerosis, could be harmful in some patients with neuromyelitis optica. In these cases natalizumab did not control or indeed increased disease activity, ${ }^{78,79}$ possibly by inhibiting macrophage but not neutrophil entry into lesions. ${ }^{80}$ Present treatments are associated with potential long-term side-effects because they do not target the cause of neuromyelitis optica, but produce general immunosuppression.

\section{Emerging treatments}

New treatments for neuromyelitis optica have been proposed that target specific components of disease pathogenesis (figure 3). Because AQP4-IgG binding to AQP4 is probably the initiating event in neuromyelitis optica, a blocker strategy has been introduced in which a non-pathogenic antibody or small molecule binds to AQP4, preventing the binding of pathogenic AQP4-IgG. ${ }^{57}$ In a proof-of-concept study, ${ }^{57}$ investigators generated a nonpathogenic, high-affinity monoclonal antibody (called aquaporumab) from a recombinant monoclonal neuromyelitis optica antibody with tight binding and slow washout (derived from clonally expanded plasmablasts in the CSF of patients) by mutation of its $\mathrm{Fc}$ region to eliminate effector functions of complement-mediated cytotoxicity and antibody-dependent cell-mediated cytotoxicity. ${ }^{57}$ Aquaporumab prevented binding of AQP4-IgG in the sera of patients with neuromyelitis optica. Additionally, it prevented cytotoxicity in AQP4expressing cell cultures, in spinal cord slice cultures ex vivo, and in mice receiving intraparenchymal AQP4-IgG and complement in vivo. Aquaporumab, like other AQP4-IgG antibodies, does not alter AQP4 water permeability (unpublished).

In an alternative approach, high-throughput screening identified several small-molecule drugs and natural products that bind to AQP4 and reduce AQP4-IgG binding. ${ }^{11}$ The challenge in blocker treatment will be maintenance of therapeutic blocker concentrations in the CNS to chronically inhibit binding.

Neutrophils are important causes of CNS injury in early neuromyelitis-optica-like lesions in mice. ${ }^{66,67}$ Live neutrophils and purified neutrophil elastase also exacerbated these lesions in an ex-vivo spinal cord model. ${ }^{72}$ Sivelestat, a potent neutrophil elastase inhibitor, reduced the lesions in mice and ex vivo by inhibiting neutrophil entry into the lesion and tissue damage produced by neutrophil elastase. Sivelestat is licensed in Japan for acute respiratory distress syndrome, but is not approved in Europe or the USA. Assessment of sivelestat as a shortterm corticosteroid-sparing agent to treat acute exacerbations of neuromyelitis optica is warranted. 


\section{Future directions}

Improved animal models are needed to further elucidate neuromyelitis optica pathogenesis and to test new treatments. The next generation of models might use active immunisation to study the initial immune response against AQP4, the generation of AQP4-IgG, and its entry into the CNS. Although rodents have been used for passive transfer, they might not be an appropriate species for active immunisation. The amount of brain tissue made up of glial cells is $90 \%$ in people and $65 \%$ in mice. ${ }^{112}$ Mice also have lower neutrophil counts than do people (5-10\% vs 60-70\%), and a poorer responsive complement system. ${ }^{113}$

\section{Search strategy and selection criteria}

References for this report were identified through searches of PubMed; the ECTRIMS website; and the websites of Annals of Neurology, Neurology, Brain, and Multiple Sclerosis. The search terms used were "AQP4", "aquaporin-4", "neuromyelitis optica", and "Devic's disease". Reports in English published between Jan 1, 1970, and April 5, 2012 , were reviewed. References were chosen on the basis of originality and relevance to the broad scope of this Review.

Although many anecdotal reports and open-label studies suggest that immunosuppressive treatments and plasmapheresis are effective in neuromyelitis optica, few well controlled clinical trials have been done. The small number of patients and their highly variable clinical course (partly a result of the present diagnostic system) will remain major challenges in trials. New treatments targeting AQP4-IgG binding to AQP4 are promising. Results of an open-label trial (NCT00904826) of the complement inhibitor eculizumab in refractory neuromyelitis optica are eagerly awaited, not withstanding the drug's high cost (US\$400 000 per patient per year) and risk of meningococcal meningitis and other infections. The repurposing of existing drugs, such as the neutrophil inhibitor sivelestat and the IL-6 receptor inhibitor tocilizumab, for treatment of neuromyelitis optica also warrants consideration. In the long term, targeted immunomodulation-perhaps tolerising treatment -might be sufficiently advanced to warrant possible use in neuromyelitis optica.

\section{Acknowledgments}

MCP and ASV were supported by the Guthy-Jackson Charitable Foundation; ASV was also supported by the National Institutes of Health. The funding sources were not involved in writing the manuscript or the decision to submit for publication. We thank B Weinshenker (Mayo Clinic) and S Zamvil (University of California San Francisco) for critical review of this report.

\section{References}

1. Jarius S, Wildemann B. 'Noteomielite' accompanied by acute amaurosis (1844): an early case of neuromyelitis optica. J Neurol Sci. 2012; 313:182-84. [PubMed: 21992815]

2. Jarius S, Wildemann B. The case of the Marquis de Causan (1804): an early account of visual loss associated with spinal cord inflammation. J Neurol Sci. 2012; 313:182-84. [PubMed: 21992815]

3. Jarius S, Wildemann B. An early case of neuromyelitis optica: on a forgotten report by Jacob Lockhart Clarke, FRS. Mult Scler. 2011; 17:1384-86. [PubMed: 21690148]

4. Lennon VA, Wingerchuk DM, Kryzer TJ, et al. A serum autoantibody marker of neuromyelitis optica: distinction from multiple sclerosis. Lancet. 2004; 364:2106-12. [PubMed: 15589308]

5. Lennon VA, Kryzer TJ, Pittock SJ, Verkman AS, Hinson SR. IgG marker of optic-spinal multiple sclerosis binds to the aquaporin-4 water channel. J Exp Med. 2005; 202:473-77. [PubMed: 16087714]

6. Collongues N, de Seze J. Current and future treatment approaches for neuromyelitis optica. Ther Adv Neurol Disord. 2011; 4:111-21. [PubMed: 21694808] 
7. Wingerchuk DM, Lennon VA, Pittock SJ, Lucchinetti CF, Weinshenker BG. Revised diagnostic criteria for neuromyelitis optica. Neurology. 2006; 66:1485-89. [PubMed: 16717206]

8. Wingerchuk DM, Lennon VA, Lucchinetti CF, Pittock SJ, Weinshenker BG. The spectrum of neuromyelitis optica. Lancet Neurol. 2007; 6:805-15. [PubMed: 17706564]

9. Mader S, Lutterotti A, Di Pauli F, et al. Patterns of antibody binding to aquaporin-4 isoforms in neuromyelitis optica. PLoS One. 2010; 5:e10455. [PubMed: 20463974]

10. Bourre B, Marignier R, Zephir H, et al. Neuromyelitis optica and pregnancy. Neurology. 2012; 78:875-79. [PubMed: 22402855]

11. Gomori E, Pal J, Abraham H, et al. Fetal development of membrane water channel proteins aquaporin-1 and aquaporin-4 in the human brain. Int J Dev Neurosci. 2006; 24:295-305. [PubMed: 16814974]

12. Jarius S, Paul F, Franciotta D, et al. Neuromyelitis optica spectrum disorders in patients with myasthenia gravis: ten new aquaporin-4 antibody positive cases and a review of the literature. Mult Scler. 2011 published online Dec 19. 10.1177/1352458511431728

13. McKeon A, Lennon VA, Jacob A, et al. Coexistence of myasthenia gravis and serological markers of neurological autoimmunity in neuromyelitis optica. Muscle Nerve. 2009; 39:87-90. [PubMed: 19086079]

14. Matiello M, Kim HJ, Kim W, et al. Familial neuromyelitis optica. Neurology. 2010; 75:310-15. [PubMed: 20660861]

15. Zephir H, Fajardy I, Outteryck O, et al. Is neuromyelitis optica associated with human leukocyte antigen? Mult Scler. 2009; 15:571-79. [PubMed: 19299434]

16. Wang H, Dai Y, Qiu W, et al. HLA-DPB1 0501 is associated with susceptibility to antiaquaporin-4 antibodies positive neuromyelitis optica in southern Han Chinese. J Neuroimmunol. 2011; 233:181-84. [PubMed: 21130504]

17. Matiello M, Schaefer-Klein JL, Hebrink DD, Kingsbury DJ, Atkinson EJ, Weinshenker BG. Genetic analysis of aquaporin-4 in neuromyelitis optica. Neurology. 2011; 77:1149-55. [PubMed: 21900637]

18. Crane JM, Rossi A, Gupta T, Bennett JL, Verkman AS. Orthogonal array formation by human aquaporin-4: examination of neuromyelitis optica-associated aquaporin-4 polymorphisms. J Neuroimmunol. 2011; 236:93-98. [PubMed: 21621278]

19. Hasegawa H, Ma T, Skach W, Matthay MA, Verkman AS. Molecular cloning of a mercurialinsensitive water channel expressed in selected water-transporting tissues. J Biol Chem. 1994; 269:5497-500. [PubMed: 7509789]

20. Ho JD, Yeh R, Sandstrom A, et al. Crystal structure of human aquaporin 4 at $1.8 \mathrm{~A}$ and its mechanism of conductance. Proc Natl Acad Sci USA. 2009; 106:7437-42. [PubMed: 19383790]

21. Wolburg H, Wolburg-Buchholz K, Fallier-Becker P, Noell S, Mack AF. Structure and functions of aquaporin-4-based orthogonal arrays of particles. Int Rev Cell Mol Biol. 2011; 287:1-41. [PubMed: 21414585]

22. Yang B, Brown D, Verkman AS. The mercurial insensitive water channel (AQP-4) forms orthogonal arrays in stably transfected Chinese hamster ovary cells. J Biol Chem. 1996; 271:457780. [PubMed: 8617713]

23. Verbavatz JM, Ma T, Gobin R, Verkman AS. Absence of orthogonal arrays in kidney, brain and muscle from transgenic knockout mice lacking water channel aquaporin-4. J Cell Sci. 1997; 110:2855-60. [PubMed: 9427293]

24. Crane JM, Van Hoek AN, Skach WR, Verkman AS. Aquaporin-4 dynamics in orthogonal arrays in live cells visualized by quantum dot single particle tracking. Mol Biol Cell. 2008; 19:3369-78. [PubMed: 18495865]

25. Crane JM, Verkman AS. Determinants of aquaporin-4 assembly in orthogonal arrays revealed by live-cell single-molecule fluorescence imaging. J Cell Sci. 2009; 122:813-21. [PubMed: 19240114]

26. Crane JM, Bennett JL, Verkman AS. Live cell analysis of aquaporin- $4 \mathrm{~m} 1 / \mathrm{m} 23$ interactions and regulated orthogonal array assembly in glial cells. J Biol Chem. 2009; 284:35850-60. [PubMed: 19843522] 
27. Crane JM, Lam C, Rossi A, Gupta T, Bennett JL, Verkman AS. Binding affinity and specificity of neuromyelitis optica autoantibodies to aquaporin-4 M1/M23 isoforms and orthogonal arrays. $\mathrm{J}$ Biol Chem. 2011; 286:16516-24. [PubMed: 21454592]

28. Phuan PW, Ratelade J, Rossi A, Tradtrantip L, Verkman AS. Complement-dependent cytotoxicity in neuromyelitis optica requires aquaporin-4 assembly in orthogonal arrays. J Biol Chem. 2012; 287:13829-39. [PubMed: 22393049]

29. Furman CS, Gorelick-Feldman DA, Davidson KG, et al. Aquaporin-4 square array assembly: opposing actions of M1 and M23 isoforms. Proc Natl Acad Sci USA. 2003; 100:13609-14. [PubMed: 14597700]

30. Jin BJ, Rossi A, Verkman AS. Model of aquaporin-4 supramolecular assembly in orthogonal arrays based on heterotetrameric association of M1-M23 isoforms. Biophys J. 2011; 100:2936-45. [PubMed: 21689527]

31. Verkman AS. Aquaporins in clinical medicine. Annu Rev Med. 2012; 63:303-16. [PubMed: 22248325]

32. Rash JE, Yasumura T, Hudson CS, Agre P, Nielsen S. Direct immunogold labeling of aquaporin-4 in square arrays of astrocyte and ependymocyte plasma membranes in rat brain and spinal cord. Proc Natl Acad Sci USA. 1998; 95:11981-86. [PubMed: 9751776]

33. Nielsen S, Nagelhus EA, Amiry-Moghaddam M, Bourque C, Agre P, Ottersen OP. Specialized membrane domains for water transport in glial cells: high-resolution immunogold cytochemistry of aquaporin-4 in rat brain. J Neurosci. 1997; 17:171-80. [PubMed: 8987746]

34. Li J, Patil RV, Verkman AS. Mildly abnormal retinal function in transgenic mice without Muller cell aquaporin-4 water channels. Invest Ophthalmol Vis Sci. 2002; 43:573-79. [PubMed: 11818406]

35. Ma T, Yang B, Gillespie A, Carlson EJ, Epstein CJ, Verkman AS. Generation and phenotype of a transgenic knockout mouse lacking the mercurial-insensitive water channel aquaporin-4. J Clin Invest. 1997; 100:957-62. [PubMed: 9276712]

36. Yang B, Verbavatz JM, Song Y, et al. Skeletal muscle function and water permeability in aquaporin-4 deficient mice. Am J Physiol Cell Physiol. 2000; 278:C1108-15. [PubMed: 10837338]

37. Li J, Verkman AS. Impaired hearing in mice lacking aquaporin-4 water channels. J Biol Chem. 2001; 276:31233-37. [PubMed: 11406631]

38. Lu DC, Zhang H, Zador Z, Verkman AS. Impaired olfaction in mice lacking aquaporin-4 water channels. FASEB J. 2008; 22:3216-23. [PubMed: 18511552]

39. Manley GT, Fujimura M, Ma T, et al. Aquaporin-4 deletion in mice reduces brain edema after acute water intoxication and ischemic stroke. Nat Med. 2000; 6:159-63. [PubMed: 10655103]

40. Papadopoulos MC, Manley GT, Krishna S, Verkman AS. Aquaporin-4 facilitates reabsorption of excess fluid in vasogenic brain edema. FASEB J. 2004; 18:1291-93. [PubMed: 15208268]

41. Bloch O, Auguste KI, Manley GT, Verkman AS. Accelerated progression of kaolin-induced hydrocephalus in aquaporin-4-deficient mice. J Cereb Blood Flow Metab. 2006; 26:1527-37. [PubMed: 16552421]

42. Saadoun S, Bell BA, Verkman AS, Papadopoulos MC. Greatly improved neurological outcome after spinal cord compression injury in AQP4-deficient mice. Brain. 2008; 131:1087-98. [PubMed: 18267965]

43. Kimura A, Hsu M, Seldin M, Verkman AS, Scharfman HE, Binder DK. Protective role of aquaporin-4 water channels after contusion spinal cord injury. Ann Neurol. 2010; 67:794-801. [PubMed: 20517941]

44. Binder DK, Yao X, Zador Z, Sick TJ, Verkman AS, Manley GT. Increased seizure duration and slowed potassium kinetics in mice lacking aquaporin-4 water channels. Glia. 2006; 53:631-36. [PubMed: 16470808]

45. Saadoun S, Papadopoulos MC, Watanabe H, Yan D, Manley GT, Verkman AS. Involvement of aquaporin-4 in astroglial cell migration and glial scar formation. J Cell Sci. 2005; 118:5691-98. [PubMed: 16303850]

46. Li L, Zhang H, Varrin-Doyer M, Zamvil SS, Verkman AS. Proinflammatory role of aquaporin-4 in autoimmune neuroinflammation. FASEB J. 2011; 25:1556-66. [PubMed: 21257712] 
47. Nicchia GP, Mastrototaro M, Rossi A, et al. Aquaporin-4 orthogonal arrays of particles are the target for neuromyelitis optica autoantibodies. Glia. 2009; 57:1363-73. [PubMed: 19229993]

48. Hinson SR, Romero MF, Popescu BF, et al. Molecular outcomes of neuromyelitis optica (NMO)IgG binding to aquaporin-4 in astrocytes. Proc Natl Acad Sci USA. 2011; 109:1245-50. [PubMed: 22128336]

49. Bennett JL, Lam C, Kalluri SR, et al. Intrathecal pathogenic anti-aquaporin-4 antibodies in early neuromyelitis optica. Ann Neurol. 2009; 66:617-29. [PubMed: 19938104]

50. Pisani F, Mastrototaro M, Rossi A, et al. Identification of two major conformational aquaporin-4 epitopes for neuromyelitis optica autoantibody binding. J Biol Chem. 2011; 286:9216-24. [PubMed: 21212277]

51. Yu X, Green M, Gilden D, Lam C, Bautista K, Bennett JL. Identification of peptide targets in neuromyelitis optica. J Neuroimmunol. 2011; 236:65-71. [PubMed: 21621279]

52. Waters P, Vincent A. Detection of anti-aquaporin-4 antibodies in neuromyelitis optica: current status of the assays. Int MS J. 2008; 15:99-105. [PubMed: 18808744]

53. Rossi A, Ratelade J, Papadopoulos MC, Benett JL, Verkman AS. Consequences of NMO-IgG binding to aquaporin-4 in neuromyelitis optica. PNAS. (in press).

54. Hinson SR, Pittock SJ, Lucchinetti CF, et al. Pathogenic potential of IgG binding to water channel extracellular domain in neuromyelitis optica. Neurology. 2007; 69:2221-31. [PubMed: 17928579]

55. Ratelade J, Bennett JL, Verkman AS. Evidence against cellular internalization in vivo of NMO$\mathrm{IgG}$, aquaporin-4, and excitatory amino acid transporter 2 in neuromyelitis optica. J Biol Chem. 2011; 286:45156-64. [PubMed: 22069320]

56. Saadoun S, Waters P, Bell BA, Vincent A, Verkman AS, Papadopoulos MC. Intra-cerebral injection of neuromyelitis optica immunoglobulin $\mathrm{G}$ and human complement produces neuromyelitis optica lesions in mice. Brain. 2010; 133:349-61. [PubMed: 20047900]

57. Tradtrantip L, Zhang H, Saadoun S, et al. Anti-aquaporin-4 monoclonal antibody blocker therapy for neuromyelitis optica. Ann Neurol. 2012; 71:314-22. [PubMed: 22271321]

58. Bennett JL, Lam C, Kalluri SR, et al. Intrathecal pathogenic anti-aquaporin-4 antibodies in early neuromyelitis optica. Ann Neurol. 2009; 66:617-29. [PubMed: 19938104]

59. Ratelade J, Zhang H, Saadoun S, Bennett JL, Papadopoulos MC, Verkman AS. Neuromyelitis optica IgG and natural killer cells produce NMO lesions in mice without myelin loss. Acta Neuropathol. 2012 published online April 22. 10.1007/s00401-012-0986-4

60. Lucchinetti CF, Mandler RN, McGavern D, et al. A role for humoral mechanisms in the pathogenesis of Devic's neuromyelitis optica. Brain. 2002; 125:1450-61. [PubMed: 12076996]

61. Roemer SF, Parisi JE, Lennon VA, et al. Pattern-specific loss of aquaporin-4 immunoreactivity distinguishes neuromyelitis optica from multiple sclerosis. Brain. 2007; 130:1194-205. [PubMed: 17282996]

62. Misu T, Fujihara K, Kakita A, et al. Loss of aquaporin 4 in lesions of neuromyelitis optica: distinction from multiple sclerosis. Brain. 2007; 130:1224-34. [PubMed: 17405762]

63. Lucchinetti CF, Mandler RN, McGavern D, et al. A role for humoral mechanisms in the pathogenesis of Devic's neuromyelitis optica. Brain. 2002; 125:1450-61. [PubMed: 12076996]

64. Jarius S, Franciotta D, Bergamaschi R, Wildemann B, Wandinger KP. Immunoglobulin M antibodies to aquaporin-4 in neuromyelitis optica and related disorders. Clin Chem Lab Med. 2010; 48:659-63. [PubMed: 20184532]

65. Jarius S, Wildemann B. AQP4 antibodies in neuromyelitis optica: diagnostic and pathogenetic relevance. Nat Rev Neurol. 2010; 6:383-92. [PubMed: 20639914]

66. Saadoun S, Waters P, MacDonald C, et al. Neutrophil protease inhibition reduces NMO-IgGinduced damage in mouse brain. Ann Neurol. 2012; 71:323-33. [PubMed: 22374891]

67. Herges K, de Jong BA, Kolkowitz I, et al. Protective effect of an elastase inhibitor in a neuromyelitis optica-like disease driven by a peptide of myelin oligodendroglial glycoprotein. Mult Scler. 2012; 18:398-408. [PubMed: 22343184]

68. Jacob A, Saadoun S, Kitley J, et al. Detrimental role of granulocyte-colony stimulating factor in neuromyelitis optica: clinical case and histological evidence. Mult Scler. 2012 published online April 11. 10.1177/1352458512443994 
69. Kita H. Eosinophils: multifaceted biological properties and roles in health and disease. Immunol Rev. 2011; 242:161-77. [PubMed: 21682744]

70. Bradl M, Misu T, Takahashi T, et al. Neuromyelitis optica: pathogenicity of patient immunoglobulin in vivo. Ann Neurol. 2009; 66:630-43. [PubMed: 19937948]

71. Kinoshita M, Nakatsuji Y, Kimura T, et al. Neuromyelitis optica: passive transfer to rats by human immunoglobulin. Biochem Biophys Res Commun. 2009; 386:623-27. [PubMed: 19545538]

72. Zhang H, Bennett JL, Verkman AS. Ex vivo spinal cord slice model of neuromyelitis optica reveals novel immunopathogenic mechanisms. Ann Neurol. 2011; 70:943-54. [PubMed: 22069219]

73. Jarius S, Franciotta D, Paul F, et al. Cerebrospinal fluid antibodies to aquaporin-4 in neuromyelitis optica and related disorders: frequency, origin, and diagnostic relevance. J Neuroinflammation. 2010; 7:52. [PubMed: 20825655]

74. Misu T, Takano R, Fujihara K, Takahashi T, Sato S, Itoyama Y. Marked increase in cerebrospinal fluid glial fibrillar acidic protein in neuromyelitis optica: an astrocytic damage marker. J Neurol Neurosurg Psychiatry. 2009; 80:575-77. [PubMed: 19372295]

75. Jarius S, Paul F, Franciotta D, et al. Cerebrospinal fluid findings in aquaporin-4 antibody positive neuromyelitis optica: results from 211 lumbar punctures. J Neurol Sci. 2011; 306:82-90. [PubMed: 21550068]

76. Matsushita, T.; Tateishi, T.; Yonekawa, T.; Isobe, N.; Kira, JI. Increased CSF IL-17A, IL-6, CXCL8 and CXCL10 in patients with neuromyelitis optica at relapse and their clinical relevance. 5th Joint triennial congress of the European and Americas Committees for Treatment and Research in Multiple Sclerosis; Amsterdam, Netherlands. Oct 19-22 2011; p. 56

77. Hendriks JJ, Teunissen CE, de Vries HE, Dijkstra CD. Macrophages and neurodegeneration. Brain Res Rev. 2005; 48:185-95. [PubMed: 15850657]

78. Kleiter, I.; Hellwig, K.; Berthele, A., et al. Severe exacerbations of neuromyelitis optica during natalizumab therapy. ECTRIMS 2011 meeting; Oct 19-22 2011; p. 660

79. Barnett M, Prineas J, Buckland M, Parratt J, Pollard J. Massive astrocyte destruction in neuromyelitis optica despite natalizumab therapy. Mult Scler. 2012; 18:108-12. [PubMed: 21868485]

80. Kirveskari J, Bono P, Granfors K, Leirisalo-Repo M, Jalkanen S, Salmi M. Expression of alpha4integrins on human neutrophils. J Leukoc Biol. 2000; 68:243-50. [PubMed: 10947069]

81. Marignier R, Nicolle A, Watrin C, et al. Oligodendrocytes are damaged by neuromyelitis optica immunoglobulin G via astrocyte injury. Brain. 2010; 133:2578-91. [PubMed: 20688809]

82. Jarius S, Ruprecht K, Wildemann B, et al. Contrasting disease patterns in seropositive and seronegative neuromyelitis optica: a multicentre study of 175 patients. J Neuroinflammation. 2012; 9:14. [PubMed: 22260418]

83. McKeon A, Pittock SJ, Lennon VA. CSF complements serum for evaluating paraneoplastic antibodies and NMO-IgG. Neurology. 2011; 76:1108-10. [PubMed: 21422462]

84. Waters PJ, McKeon A, Leite MI, et al. Serologic diagnosis of NMO: a multicenter comparison of aquaporin-4-IgG assays. Neurology. 2012; 78:665-71. [PubMed: 22302543]

85. Mader S, Gredler V, Schanda K, et al. Complement activating antibodies to myelin oligodendrocyte glycoprotein in neuromyelitis optica and related disorders. J Neuroinflammation. 2011; 8:184. [PubMed: 22204662]

86. Nishiyama S, Ito T, Misu T, et al. A case of NMO seropositive for aquaporin-4 antibody more than 10 years before onset. Neurology. 2009; 72:1960-61. [PubMed: 19487655]

87. Apiwattanakul M, Popescu BF, Matiello M, et al. Intractable vomiting as the initial presentation of neuromyelitis optica. Ann Neurol. 2010; 68:757-61. [PubMed: 21031587]

88. Iorio R, Lucchinetti CF, Lennon VA, et al. Syndrome of inappropriate antidiuresis may herald or accompany neuromyelitis optica. Neurology. 2011; 77:1644-46. [PubMed: 21998320]

89. Hofman P, Hoyng P, vanderWerf F, Vrensen GF, Schlingemann RO. Lack of blood-brain barrier properties in microvessels of the prelaminar optic nerve head. Invest Ophthalmol Vis Sci. 2001; 42:895-901. [PubMed: 11274064]

90. Bartanusz V, Jezova D, Alajajian B, Digicaylioglu M. The blood-spinal cord barrier: morphology and clinical implications. Ann Neurol. 2011; 70:194-206. [PubMed: 21674586] 
91. Koga M, Takahashi T, Kawai M, Fujihara K, Kanda T. A serological analysis of viral and bacterial infections associated with neuromyelitis optica. J Neurol Sci. 2011; 300:19-22. [PubMed: 21056429]

92. Wingerchuk DM, Hogancamp WF, O’Brien PC, Weinshenker BG. The clinical course of neuromyelitis optica (Devic's syndrome). Neurology. 1999; 53:1107-14. [PubMed: 10496275]

93. Roopenian DC, Akilesh S. FcRn: the neonatal Fc receptor comes of age. Nat Rev Immunol. 2007; 7:715-25. [PubMed: 17703228]

94. Oshio K, Binder DK, Yang B, Schecter S, Verkman AS, Manley GT. Expression of aquaporin water channels in mouse spinal cord. Neuroscience. 2004; 127:685-93. [PubMed: 15283967]

95. Chihara N, Aranami T, Sato W, et al. Interleukin 6 signaling promotes anti-aquaporin 4 autoantibody production from plasmablasts in neuromyelitis optica. Proc Natl Acad Sci USA. 2011; 108:3701-06. [PubMed: 21321193]

96. Ratelade J, Bennett JL, Verkman AS. Intravenous neuromyelitis optica autoantibody in mice targets aquaporin-4 in peripheral organs and area postrema. PLoS One. 2011; 6:e27412. [PubMed: 22076159]

97. Suzuki N, Takahashi T, Aoki M, et al. Neuromyelitis optica preceded by hyperCKemia episode. Neurology. 2010; 74:1543-45. [PubMed: 20458072]

98. Ma T, Yang B, Verkman AS. Gene structure, cDNA cloning, and expression of a mouse mercurialinsensitive water channel. Genomics. 1996; 33:382-88. [PubMed: 8660998]

99. Zipfel PF, Skerka C. Complement regulators and inhibitory proteins. Nat Rev Immunol. 2009; 9:729-40. [PubMed: 19730437]

100. Faulkner JR, Herrmann JE, Woo MJ, Tansey KE, Doan NB, Sofroniew MV. Reactive astrocytes protect tissue and preserve function after spinal cord injury. J Neurosci. 2004; 24:2143-55. [PubMed: 14999065]

101. Wasseff SK, Scherer SS. Cx32 and Cx47 mediate oligodendrocyte:astrocyte and oligodendrocyte:oligodendrocyte gap junction coupling. Neurobiol Dis. 2011; 42:506-13. [PubMed: 21396451]

102. Hinson SR, Roemer SF, Lucchinetti CF, et al. Aquaporin-4-binding autoantibodies in patients with neuromyelitis optica impair glutamate transport by down-regulating EAAT2. J Exp Med. 2008; 205:2473-81. [PubMed: 18838545]

103. Auguste KI, Jin S, Uchida K, et al. Greatly impaired migration of implanted aquaporin-4deficient astroglial cells in mouse brain toward a site of injury. FASEB J. 2007; 21:108-16. [PubMed: 17135365]

104. Takahashi T, Fujihara K, Nakashima I, et al. Anti-aquaporin-4 antibody is involved in the pathogenesis of NMO: a study on antibody titre. Brain. 2007; 130:1235-43. [PubMed: 17449477]

105. Saadoun S, Waters P, Macdonald C, et al. T cell deficiency does not reduce lesions in mice produced by intracerebral injection of NMO-IgG and complement. J Neuroimmunol. 2011; 235:27-32. [PubMed: 21492943]

106. Min JH, Kim BJ, Lee KH. Development of extensive brain lesions following fingolimod (FTY720) treatment in a patient with neuromyelitis optica spectrum disorder. Mult Scler. 2012; 18:113-15. [PubMed: 22146605]

107. Nelson PA, Khodadoust M, Prodhomme T, et al. Immunodominant T cell determinants of aquaporin-4, the autoantigen associated with neuromyelitis optica. PLoS One. 2010; 5:e15050. [PubMed: 21151500]

108. Palace J, Leite MI, Nairne A, Vincent A. Interferon beta treatment in neuromyelitis optica: increase in relapses and aquaporin 4 antibody titers. Arch Neurol. 67:1016-17. [PubMed: 20697055]

109. Tanaka M, Tanaka K, Komori M. Interferon-beta(1b) treatment in neuromyelitis optica. Eur Neurol. 2009; 62:167-70. [PubMed: 19590215]

110. Krumbholz M, Faber H, Steinmeyer F, et al. Interferon-beta increases BAFF levels in multiple sclerosis: implications for B cell autoimmunity. Brain. 2008; 131:1455-63. [PubMed: 18474519] 
111. Tradtrantip L, Zhang H, Anderson MO, et al. Small-molecule inhibitors of NMO-IgG binding to aquaporin-4 reduce astrocyte cytotoxicity in neuromyelitis optica. FASEB J. 2012 published online Feb 8. 10.1096/fj.11-201608

112. Allen NJ, Barres BA. Neuroscience: glia-more than just brain glue. Nature. 2009; 457:675-77. [PubMed: 19194443]

113. von Vietinghoff S, Ley K. Homeostatic regulation of blood neutrophil counts. J Immunol. 2008; 181:5183-88. [PubMed: 18832668] 


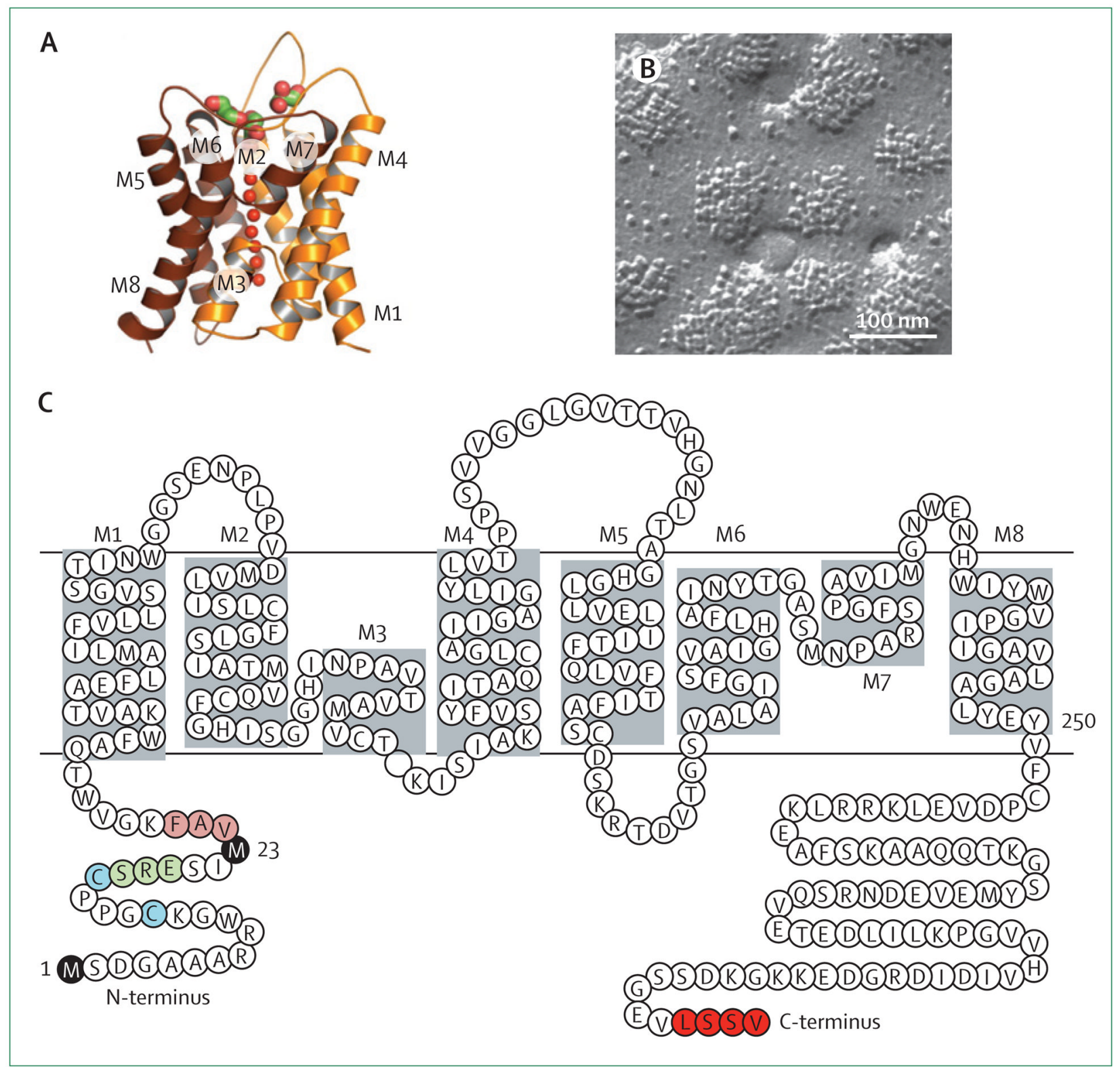

Figure 1. The astrocyte water channel AQP4

(A) High-resolution crystal structure of human AQP4 (Protein Data Bank reference 3GD8) showing eight membrane-associated helical segments, labelled M1-M8. (B) Freeze-fracture electron microscopy of AQP4 orthogonal arrays of particles in plasma membranes of Chinese hamster ovary cells transfected with M23-AQP4. (C) Protein sequence of human AQP4, showing Met-1 and Met-23 translation inhibition sites (black), residues that form intermolecular $\mathrm{N}$-terminus associations to produce orthogonal arrays of particles (pink), residues preventing formation of orthogonal arrays of particles by M1-AQP4 (green), cysteine residues involved in palmitolyation-regulated assembly of orthogonal arrays of particles (blue), and the C-terminus PDZ domain (red). 

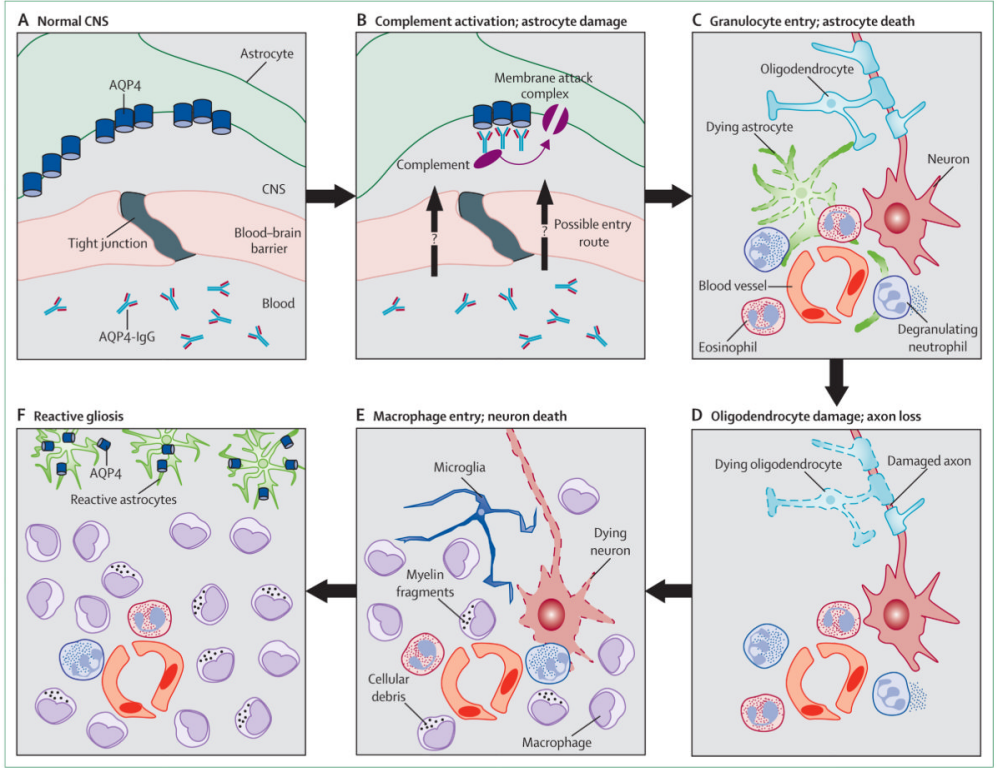

Figure 2. Formation of neuromyelitis optica lesion

(A) Normal CNS with AQP4-IgG in the serum. (B) At the start of lesion formation, AQP4IgG binds to AQP4 on foot processes of astrocytes, activates complement, and causes deposition of membrane attack complexes. (C) Cytokines (eg, interleukin 17, interleukin 8, and granulocyte colony-stimulating factor) recruit neutrophils and eosinophils into perivascular spaces; neutrophils degranulate to cause astrocyte death. Loss of astrocytes leads to oligodendrocyte death, which causes axon degeneration (D), and neuronal death (E). Infiltrating macrophages (and possibly microglia) phagocytose cellular and myelin debris. (F) Mature lesions are characterised by pan-necrosis (complete tissue necrosis), and widespread infiltration by macrophages; AQP4-positive reactive astrocytes are confined around the lesion. 


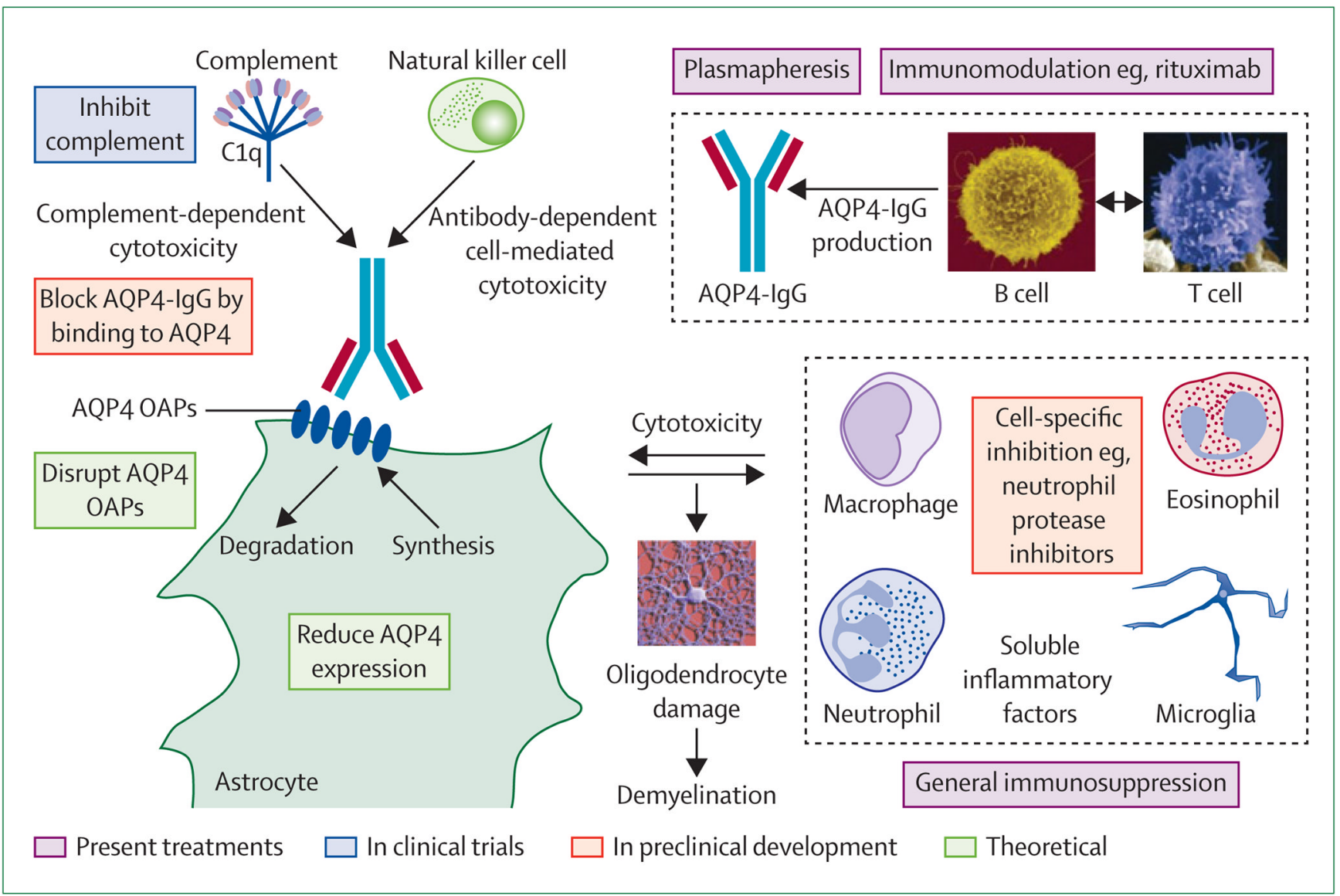

Figure 3. Potential targets for treatment

$\mathrm{OAP}=$ orthogonal array of particles. 